\title{
ANÁLISE DA INFLUÊNCIA DE DIFERENTES TAMANHOS E COMPOSIÇÕES DE AMOSTRAS NO AJUSTE DE UMA RELAÇÃO HIPSOMÉTRICA PARA Eucalyptus grandis ${ }^{1}$
}

Gilson Fernandes da Silva ${ }^{2}$, Alexandre Cândido Xavier $^{2}$, Flávio Lopes Rodrigues² e Luiz Alexandre Peternelli ${ }^{3}$

\begin{abstract}
RESUMO - O ajuste de relações hipsométricas a partir de dados oriundos de inventário florestal com o objetivo de estimar o volume de árvores individuais é procedimento comum nas empresas florestais brasileiras. Assim, considerando um conjunto de 481 árvores de Eucalyptus grandis, obtido por meio de um procedimento de inventário florestal, foram simulados diversos tamanhos de amostra, variando de 6 a 204 árvores-amostra. Para cada tamanho de amostra foram feitas 10.000 simulações para cada tamanho de amostra obtidas aleatoriamente. Considerando o exposto, este trabalho teve como objetivo avaliar o efeito do tamanho e da composição das amostras simuladas sobre a precisão de um modelo hipsométrico previamente selecionado. O modelo selecionado teve como variáveis independentes o diâmetro à altura do peito $(D A P)$ e a idade, sendo avaliado também o efeito dessas variáveis sobre as estimativas de altura. Ao final, conclui-se que amostras com pelo menos 27 árvores proporcionaram estimativas mais precisas da altura das árvores quando comparadas com amostras menores que esse número. No entanto, amostras maiores que 27 árvores não implicaram ganhos significativos em termos de precisão. Conclui-se também que a variável $D A P$ foi mais importante para estimar a altura do que a variável idade, considerando-se o modelo proposto, sendo a influência da variável idade na estimação da altura maior nas árvores de maior $D A P$.
\end{abstract}

Palavras-chave: Altura da árvore, amostragem e inventário florestal.

\section{EVALUATION OF THE INFLUENCE OF VARYING SAMPLE SIZES AND COMPOSITION ON THE PRECISION OF A HYPSOMETRIC RELATION FOR Eucalyptus grandis}

\begin{abstract}
The adjustment of hypsometric relations using data from forest inventory aiming at the estimation of individual tree volumes is a common task in Brazilian forestry companies. This study used a data set of 481 trees of Eucalyptus grandis from a forest inventory. Simulation was carried out varying sample sizes from 6 to 204 sample-trees. For each sample size it was run 10,000 simulations for each size samples selected randomly. This study aimed at evaluating the effect of sample size and structure on the precision of a previously chosen hypsometric model which had, as independent variables, the Diameter at Breast Height (DBH) and the tree age. It was also evaluated the effect of these covariates on the tree heights estimates. It was concluded that a sample with at least 27 trees could more precisely estimate tree height when compared to smaller samples. In addition, sample sizes containing more than 27 trees did not shown significant improving on the precision of the height estimates. For the hipysometric model, the variable DBH was more valuable to estimate the tree height than the covariate age. For the trees with larger DBH, the variable age showed to be more influent.
\end{abstract}

Keywords: Tree height, sampling and forest inventory.

\footnotetext{
${ }^{1}$ Recebido em 03.01.2006 e aceito para publicação em 29.03.2007

${ }^{2}$ Centro de Ciências Agrárias da Universidade Federal do Espírito Santo, Alto Universitário, Cx. P. 16, 29500-000 AlegreES.E-mail:<gfsilva@cca.ufes.br>.

${ }^{3}$ Departamento de Informática da Universidade Federal de Viçosa (UFV), 36570-000 Viçosa-MG.
} 


\section{INTRODUÇÃO}

É necessidade constante das empresas florestais desenvolver metodologias para estimar a produção de volume ou biomassa de madeira, seja para o planejamento da produção ou para a definição de estratégias de sua comercialização. A estimativa de produção de madeira em florestas normalmente é feita por procedimentos de inventário florestal, e, para estimar o volume, busca-se relacionar variáveis como o diâmetro à altura do peito $(D A P)$ e a altura total da árvore com o seu volume. Na operacionalização do inventário, o DAP é uma variável de fácil obtenção, o que não ocorre com a altura.

De acordo com Couto e Bastos (1986), a determinação da altura das árvores em pé por meio de instrumentos é uma operação onerosa e sujeita a erros. Em razão disso, o que se tem feito na prática é medir a altura de algumas árvores nas parcelas de inventário e, empregando relações hipsométricas, estimar a altura das demais. Esse tipo de procedimento é muito comum nas espécies florestais cultivadas, como são os casos das culturas de Eucalytpus e Pinus no Brasil. Nesses gêneros, vários modelos estatísticos já foram testados e são correntemente utilizados pelas empresas florestais do país.

Ao se empregarem relações hipsométricas para estimar a altura das árvores, alguns cuidados, no entanto, devem ser tomados, sob pena de se cometerem erros grosseiros de estimação das alturas. Conforme citado por vários autores, as relações hipsométricas são afetadas por diversos fatores, podendo-se citar a idade, a qualidade do local, a densidade, posição sociológica e as práticas silviculturais (LOETSCH et al., 1973; FINGER, 1992; SCOLFORO, 1993; BATISTA et al., 1986). Assim, devese ter um cuidado especial na escolha das árvores que comporão a amostra a ser utilizada no ajuste da relação hipsométrica. Tendo em vista os muitos fatores que afetam a relação hipsométrica, a composição e tamanho da amostra devem ser determinados de forma criteriosa, de modo a evitar estimativas muito distorcidas em situações específicas.

De acordo com Schneider (1986), para a determinação de uma relação hipsométrica são necessários, em média, 30 a 40 alturas distribuídas em toda a amplitude diamétrica. Nas empresas, conforme mencionado, os dados para determinação da relação hipsométrica normalmente são oriundos dos procedimentos de inventário florestal, em que se medem, em cada parcela, as alturas das 15 ou 20 primeiras árvores, reunindo-se ao final os pares de $D A P$ e altura das árvores de todas as parcelas amostradas no inventário. Com esse procedimento, espera-se que a amostra tenha tamanho suficiente e também árvores que contemplem todas as fontes de variação possíveis que possam afetar a qualidade da relação hipsométrica em termos de precisão.

Considerando o exposto, este estudo teve como objetivo principal avaliar o tamanho e a composição de uma amostra para ajuste de uma relação hipsométrica obtida por meio de um procedimento tradicional de inventário florestal. Como objetivos específicos, podem ser citados:

Comparar diferentes tamanhos de amostras, considerando-se seus efeitos sobre a precisão de um modelo hipsométrico previamente selecionado.

Por meio de simulação, avaliar diferentes composições de amostras de cada tamanho determinado, avaliando-se também os efeitos sobre a precisão do modelo hipsométrico previamente selecionado.

Avaliação da influência das variáveis DAP e idade sobre as estimativas de altura obtidas pela relação hipsométrica proposta.

\section{MATERIAL E MÉTODOS}

\subsection{Fonte dos dados}

Para realização das análises propostas neste trabalho, foram utilizados dados obtidos a partir de um inventário realizado em povoamentos de Eucalyptus grandis, localizados no Município de Dores do Turvo, MG, pertencentes a uma propriedade particular localizada aproximadamente a $20^{\circ} 55^{\prime}$ de latitude e $43^{\circ} 06^{\prime}$ de longitude. $\mathrm{O}$ sistema de amostragem utilizado foi o estratificado, sendo os estratos determinados pelas diferentes classes de idade, conforme mostrado na Tabela 1. Parcelas temporárias medindo $600 \mathrm{~m}^{2}$ foram demarcadas em cada estrato, sendo lançadas nos estratos de 60, 74 e 84 meses 7, 5 e 10 parcelas, respectivamente. 
Tabela 1 - Caracterização das áreas plantadas com Eucalyptus grandis na propriedade inventariada

Table 1 - Characteristics of stands of Eucalyptus grandis in the inventorial area

\begin{tabular}{cccc}
\hline $\begin{array}{l}\text { Número do } \\
\text { Projeto }\end{array}$ & $\begin{array}{c}\text { Área } \\
\text { (ha) }\end{array}$ & $\begin{array}{c}\text { Idade do } \\
\text { Povoamento (meses) }\end{array}$ & Estrato \\
\hline 1 & 11,58 & 60 & 1 \\
2 & 6,00 & 60 & 1 \\
3 & 11,94 & 60 & 1 \\
4 & 41,50 & 74 & 2 \\
5 & 76,41 & 84 & 3 \\
\hline
\end{tabular}

Em cada parcela, foram medidos os diâmetros do tronco à altura de $1,30 \mathrm{~m}(D A P s)$ de todas as árvores com $D A P$ superior a $5 \mathrm{~cm}$, utilizando-se, para tanto, de uma suta. As alturas totais foram medidas nas 18 primeiras árvores de cada parcela, utilizando-se um hipsômetro de Blume-Leiss. Aos pares de altura e diâmetro medidos no inventário foram somados os dados de 85 árvores cubadas rigorosamente para a obtenção de equação de volume, chegando-se ao total de 481 pares de DAP e altura total para ajuste e simulação das equações hipsométricas. A Tabela 2 ilustra a distribuição de freqüência das 481 árvores-amostra empregadas neste estudo, em classes de diâmetro e altura.

\subsection{Modelo hipsométrico}

O modelo hipsométrico utilizado para a realização das simulações foi:

$$
Y=\beta_{o}+\beta_{1} X_{1}+\beta_{2} X_{2}+\varepsilon
$$

em que: $Y=1 /(H-1,3)$, sendo $H=$ altura total da árvore, em $\mathrm{m} ; X_{1}=1 / D A P, D A P$ em cm; $X_{2}=1 / I d$, sendo $I d$ a idade da árvore, em meses; $\beta_{i}$, os parâmetros a serem estimados ( $i=0,1$ e 2$)$; e $\varepsilon=$ erro aleatório.

As estatísticas da equação ajustada para o modelo (1), considerando-se as 481 árvores amostradas, foram as seguintes:

$\frac{1}{H-1,3}=-0,02444+0,69738^{*} \frac{1}{D}+2,32867^{*} \frac{1}{I d}$

$\operatorname{com} R^{2}=0,82 ; S_{y, x}(\%)= \pm 7,93 ; \mathrm{e}^{*}$ indicando que a estimativa do parâmetro foi significativa pelo teste $t$ em nível de $5 \%$ de probabilidade.

\subsection{Simulação do ajuste de relações hipsométricas com diferentes tamanhos e composições de amostra}

Para a simulação do ajuste das relações hipsométricas com diferentes tamanhos e composições de amostra, foi desenvolvida uma rotina em ambiente MATLAB (MATHWORKS, 1996). A Figura 1 ilustra o algoritmo de simulação empregado neste trabalho.

Tabela 2 - Distribuição das 481 árvores-amostra empregadas no estudo em classes de diâmetro e altura, e tanto as classes de diâmetro e altura apresentam uma amplitude de classe igual a 2 e estão representadas pelos seus centros de classe

Table 2 -Distribution of the 481 sample-trees used in the study into diameter and height classes; both diameter and height classes had class amplitude of 2 and are represented by their class centers

\begin{tabular}{|c|c|c|c|c|c|c|c|c|c|c|c|c|c|c|c|c|}
\hline \multirow{2}{*}{$\begin{array}{l}\text { Classes de } \\
D A P(\mathrm{~cm})\end{array}$} & \multicolumn{15}{|c|}{ Classes de Altura (m) } & \multirow[t]{2}{*}{ Total } \\
\hline & 5 & 7 & 9 & 11 & 13 & 15 & 17 & 19 & 21 & 23 & 25 & 27 & 29 & 31 & 33 & \\
\hline 6 & 2 & 15 & 3 & 4 & 1 & 0 & 0 & 0 & 0 & 0 & 0 & 0 & 0 & 0 & 0 & 25 \\
\hline 8 & 1 & 3 & 18 & 13 & 4 & 4 & 0 & 0 & 0 & 0 & 0 & 0 & 0 & 0 & 0 & 43 \\
\hline 10 & 0 & 3 & 7 & 11 & 15 & 9 & 3 & 2 & 1 & 0 & 0 & 0 & 0 & 0 & 0 & 51 \\
\hline 12 & 0 & 0 & 0 & 3 & 12 & 9 & 14 & 8 & 6 & 0 & 0 & 0 & 0 & 0 & 0 & 52 \\
\hline 14 & 0 & 0 & 1 & 1 & 4 & 11 & 7 & 11 & 8 & 7 & 2 & 0 & 0 & 0 & 0 & 52 \\
\hline 16 & 0 & 0 & 0 & 0 & 0 & 2 & 5 & 11 & 12 & 12 & 4 & 2 & 0 & 0 & 0 & 48 \\
\hline 18 & 0 & 0 & 0 & 0 & 0 & 2 & 4 & 8 & 8 & 13 & 10 & 3 & 2 & 0 & 0 & 50 \\
\hline 20 & 0 & 0 & 0 & 0 & 1 & 0 & 3 & 5 & 5 & 12 & 14 & 8 & 6 & 0 & 0 & 54 \\
\hline 22 & 0 & 0 & 0 & 0 & 0 & 0 & 2 & 1 & 2 & 11 & 19 & 11 & 6 & 0 & 0 & 52 \\
\hline 24 & 0 & 0 & 0 & 0 & 0 & 0 & 0 & 1 & 0 & 6 & 3 & 8 & 6 & 1 & 0 & 25 \\
\hline 26 & 0 & 0 & 0 & 0 & 0 & 0 & 0 & 0 & 0 & 0 & 11 & 2 & 2 & 2 & 0 & 17 \\
\hline 28 & 0 & 0 & 0 & 0 & 0 & 0 & 0 & 0 & 0 & 0 & 1 & 2 & 2 & 1 & 0 & 6 \\
\hline 30 & 0 & 0 & 0 & 0 & 0 & 0 & 0 & 0 & 0 & 0 & 0 & 1 & 1 & 1 & 1 & 4 \\
\hline 32 & 0 & 0 & 0 & 0 & 0 & 0 & 0 & 0 & 0 & 0 & 0 & 0 & 1 & 0 & 1 & 2 \\
\hline Total & 3 & 21 & 29 & 32 & 37 & 37 & 38 & 47 & 42 & 61 & 64 & 37 & 26 & 5 & 2 & 481 \\
\hline
\end{tabular}




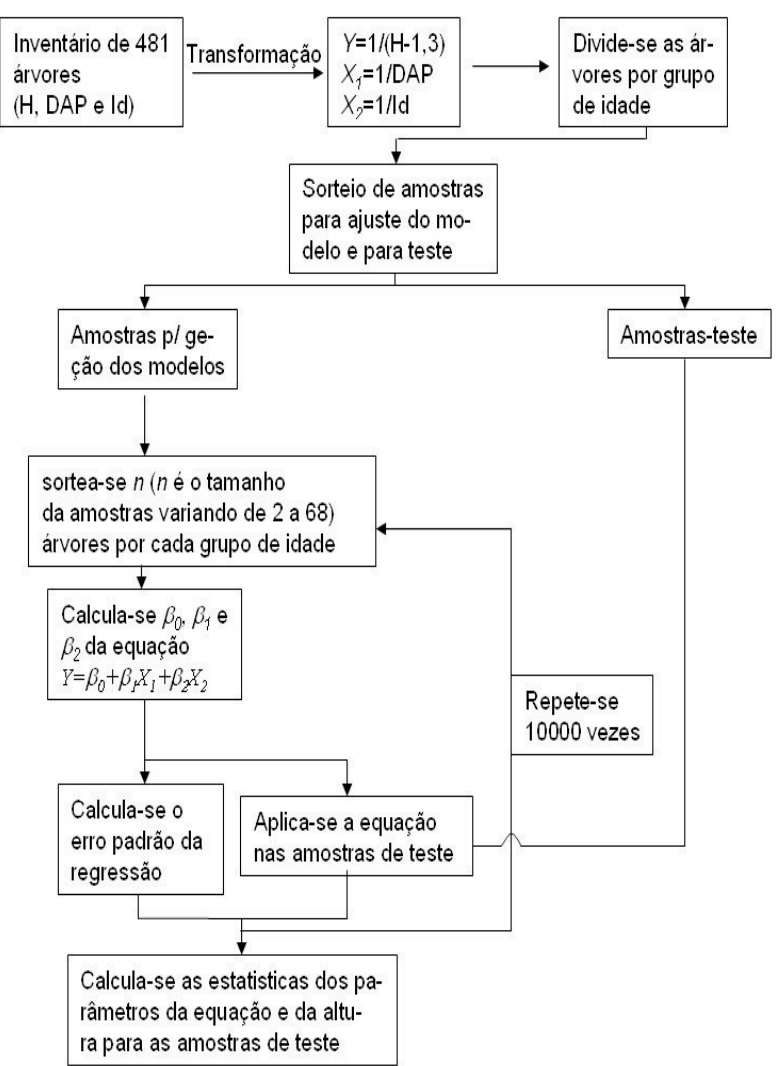

Figura 1-Descrição da metodologia empregada para a simulação. Figure 1-Description of the methodology used for the simulation.

A seguir é feita uma descrição mais detalhada da metodologia apresentada na Figura 1. Para facilitar a compreensão do leitor em relação à linguagem empregada, é importante distinguir as expressões amostra e unidade de amostra. A amostra corresponde ao conjunto de árvores empregado para ajustar uma equação específica para representar uma relação hipsométrica, sendo a equação ajustada e sempre associada ao modelo (1). No caso deste estudo, as amostras devem variar em tamanho e composição. Cada amostra, independentemente de seu tamanho ou composição, é formada por um conjunto de árvores, sendo cada árvore uma unidade de amostra ou árvore-amostra. Assim, a Figura 1 pode ser mais bem compreendida pelos seguintes passos:

i) A partir dos dados originais foram calculadas as variáveis dependentes e independentes do modelo (1) de regressão ( $Y, X_{1}$ e $\left.X_{2}\right)$ em todas as árvores; ii) os dados foram, então, agrupados por idade; iii) para cada grupo de idade foram sorteadas, aleatoriamente,
30 árvores para teste, denominadas neste trabalho amostra-teste, sendo o restante das árvores-amostra utilizadas para o ajuste das equações de regressão com diferentes tamanhos amostrais; iv) no conjunto de árvores-amostra para ajuste das equações, foram tomados aleatoriamente os dados $\left(Y, X_{1}\right.$ e $\left.X_{2}\right)$ para determinado tamanho amostral, contendo sempre igual quantidade de árvores de todas as idades, começando com duas árvores por idade, ou seja, um tamanho de amostra contendo seis árvores-amostra para ajuste do modelo (modelo (1)); v) a partir dessas amostras, a começar por seis árvores-amostra, calculavam-se as estimativas dos parâmetros do modelo pelo método dos mínimos quadrados e o erro-padrão da regressão; vi) tal equação foi aplicada a cada uma das árvores da amostra-teste para cálculo da altura estimada; vii) os procedimentos de ii a vi se repetiam mais 9.999 vezes, de forma a obter no final: a média, o desvio-padrão e o coeficiente de variação $(C V)$ dos erros-padrão da regressão, estimativa dos parâmetros e altura das árvores da amostra-teste. Os procedimentos descritos anteriormente foram repetidos para tamanhos de amostra contendo 3, 4,.., 68 árvores-amostra por grupo de idade, para se gerarem os dados (item vii).

Os resultados das simulações realizadas de acordo com a metodologia proposta foram, então, analisados em forma de gráficos, mostrando, por exemplo, as relações entre os diferentes tamanhos de amostras com: erro-padrão das equações de regressão, $C V(\%)$ das estimativas dos parâmetros do modelo proposto e a estimativa de altura das árvores contidas nas amostras-teste.

\section{RESULTADOS E DISCUSSÃO}

Na Figura 2 é apresentada a relação entre o erropadrão médio das regressões ajustadas a partir das 10.000 simulações para cada tamanho de amostra utilizado no ajuste do modelo (1). Para um tamanho de amostra pequeno, contendo seis e nove árvores-amostra, encontraram-se valores dos erros-padrão médios menores, em comparação com aqueles gerados de uma regressão com um tamanho de amostra maior. Nesse sentido, pode-se destacar o erro-padrão médio da regressão quando utilizada uma amostra com seis árvoresamostra para ajuste das equações de regressão, que foi de $\pm 1,97 \mathrm{~m}$. O erro-padrão médio chega a ser quase constante a partir de amostras com tamanho contendo 27 árvores-amostra para ajuste das equações, 
apresentando um valor aproximadamente constante $\mathrm{de} \pm 2,43 \mathrm{~m}$. Tal resultado pode ser explicado pelo reduzido número de graus de liberdade do resíduo quando se ajustaram as regressões com amostras contendo menor número de árvores-amostra, ou seja, quando se tem uma relação estreita entre o número de árvores-amostra e o número de parâmetros do modelo, há tendência de diminuição do erro-padrão da regressão. Essa diminuição não necessariamente garante que o modelo ajustado seja o mais adequado, uma vez que as amostras contempladas podem não conter a informação suficiente para representar a população-alvo. Mais adiante, tal fato será discutido em maiores detalhes.

A Figura 3 ilustra a relação entre o tamanho das amostras e o coeficiente de variação $(C V)$ calculado dos três estimadores dos parâmetros do modelo (1) a partir das 10.000 simulações geradas. Observa-se, nessa figura, que os $C V s$ calculados do coeficiente $\hat{\beta}_{1}$ foram os que apresentaram menores valores, em comparação com os coeficientes $\hat{\beta}_{0}$ e $\hat{\beta}_{2}$, e a amplitude total de variação do $C V$ para $\hat{\beta}_{1}$, considerando-se obviamente os números mínimo e máximo de árvoresamostra utilizadas para ajuste das equações, foi de aproximadamente $39 \%$, enquanto para $\hat{\beta}_{0}$ e $\hat{\beta}_{2}$ a amplitude total do $C V$ foi, respectivamente, de $179 \%$ e $131 \%$. Esse resultado indica que o coeficiente $\hat{\beta}_{1}$ foi menos afetado pelas diferentes composições e tamanhos de amostras simulados.

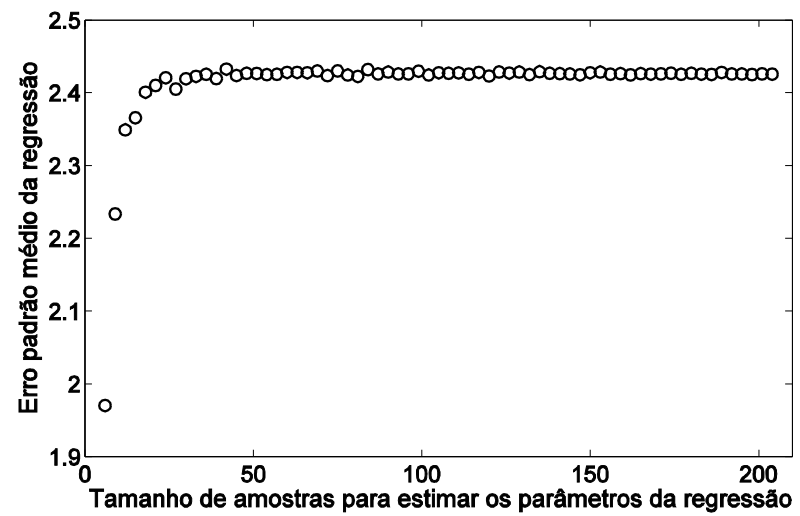

Figura 2 - Relação entre o número de árvores-amostra e o erro-padrão médio calculado a partir das equações ajustadas para o modelo (1) nas 10.000 simulações realizadas.

Figure 2 - Ratio between sample-trees and mean standard error calculated from the fitting equations adjusted from model (1) for the 10,000 simulations.

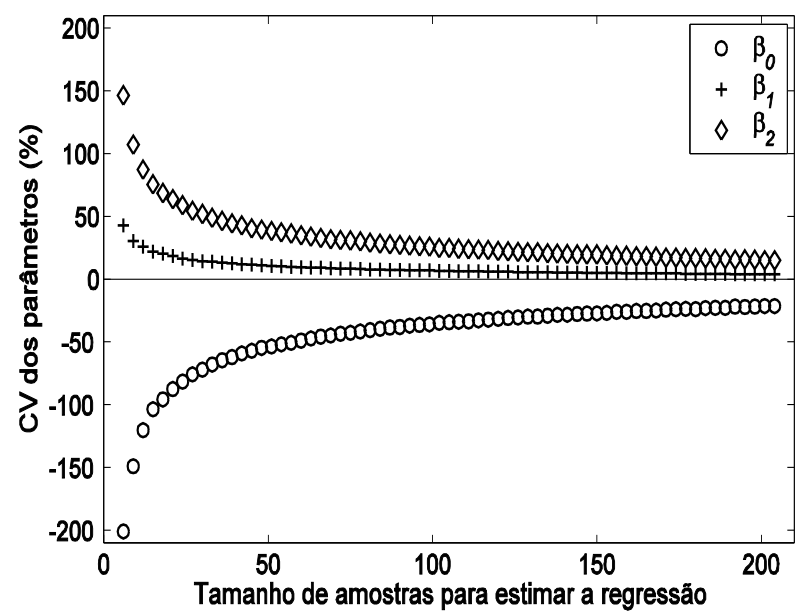

Figura 3 - Relação entre o coeficiente de variação dos estimadores dos parâmetros do modelo (1) e o tamanho das amostras consideradas.

Figure 3-Ratio between variation coefficient of the estimated parameters and the sample size used to estimate the equations from the model (1).

A taxa de diminuição dos $C V s$ é maior quando o número de árvores-amostra é pequeno, estabilizandose logo em seguida. De maneira geral, tanto para $\hat{\beta}_{0}$ quanto para $\hat{\beta}_{2}$, a estabilização do $C V$ ocorreu para um número próximo a 51 árvores-amostra, ou seja, quando se utilizaram aproximadamente 17 árvores por idade para ajustar o modelo de regressão. O $C V$ do maior número de árvores-amostra (204) chegou a $-21 \%$, $4 \%$ e $14 \%$ para $\hat{\beta}_{0}, \hat{\beta}_{1}$ e $\hat{\beta}_{2}$, respectivamente. Considerando essa variação dos $C V s$ dos estimadores dos parâmetros, um pergunta que parece ser pertinente é como esses parâmetros afetam a estimativa da altura das árvores quando utilizados no modelo (1) proposto? Uma consideração importante é o fato de que determinado estimador de parâmetro, apesar de apresentar um $C V$ elevado, pode ter impacto reduzido na estimativa de $H$ (altura), tendo em vista o modelo proposto. Na tentativa de responder a essa pergunta, considera-se a equação (2), que leva em conta todas as árvores-amostra mensuradas. Na Figura 4(a) é apresentado o resultado dessa equação, incluindo os pontos relativos a todas as árvores-amostra consideradas. Na Figura 4(b) é apresentado o campo gradiente da mesma função. A direção e magnitude das setas indicam como as variáveis independentes, $D A P$ e $I d$, estão afetando as estimativas de altura das árvores $(H)$. Considerando a direção das setas, observa-se que: i) a variável $D A P$ é a que apresenta

R. Árvore, Viçosa-MG, v.31, n.4, p.685-694, 2007 
maior influência na variação da altura das árvores, isto é, considerando-se o modelo (1), de onde se pode deduzir que variações na altura das árvores estão mais associadas ao $D A P$ do que à idade das árvores. Tal resultado pode ser observado também na Figura 4(a); ii) pequenas variações do $D A P$, em povoamentos de maior idade, implicam maior variação do valor da altura das árvores; iii) a variável Id só terá alguma contribuição mais significativa na variação da altura para maiores valores de $D A P$. Isso significa, neste caso, que não considerar a variável idade no modelo hipsométrico redundaria em maiores erros na estimação da altura das maiores árvores. Considerando que as maiores árvores possuem maior volume, esses erros podem ser propagados na determinação do volume de forma mais significativa, ressaltando-se a importância da variável idade no caso desse modelo.

Quando esses resultados são analisados concomitantemente com os resultados dos $C V s$ dos parâmetros da simulação (Figura 3), verifica-se que o estimador de parâmetro que teve menor $C V$ em todos os tamanhos de amostra foi o $\hat{\beta}_{1}$ (Figura 3 ), sendo esse o parâmetro relacionado com a variável $D A P$ no modelo (1). Esse resultado pode ser considerado favorável, quando se pensa na precisão do modelo de regressão, tendo em vista que o $D A P$ é a variável dendrométrica que mais afetou as variações na estimativa da altura. Espera-se ainda que equações oriundas de diferentes tamanhos de amostra apresentem a mesma tendência, ou seja, de que o $D A P$ seja a variável mais importante para a estimação da altura.

Uma preocupação neste trabalho foi avaliar a aplicação das equações ajustadas a partir de diferentes composições de amostras simuladas em árvores que não participaram do ajuste das regressões (árvoresteste, sendo 30 por idade). Nesse sentido, conforme descrito na metodologia, foram determinados tamanhos de amostra a partir de seis árvores (duas para cada idade) até 204 árvores. Em cada tamanho de amostra determinado foram feitas 10.000 simulações de composição da amostra, resultando em 10.000 respectivas equações de regressão. Em cada regressão simulada, foi calculado o valor estimado da altura das árvoresteste. Em uma mesma árvore-teste, calculou-se, então o $C V$ das 10.000 alturas estimadas a partir das 10.000 equações geradas, tudo isso repetido para cada tamanho de amostra.

R. Árvore, Viçosa-MG, v.31, n.4, p.685-694, 2007

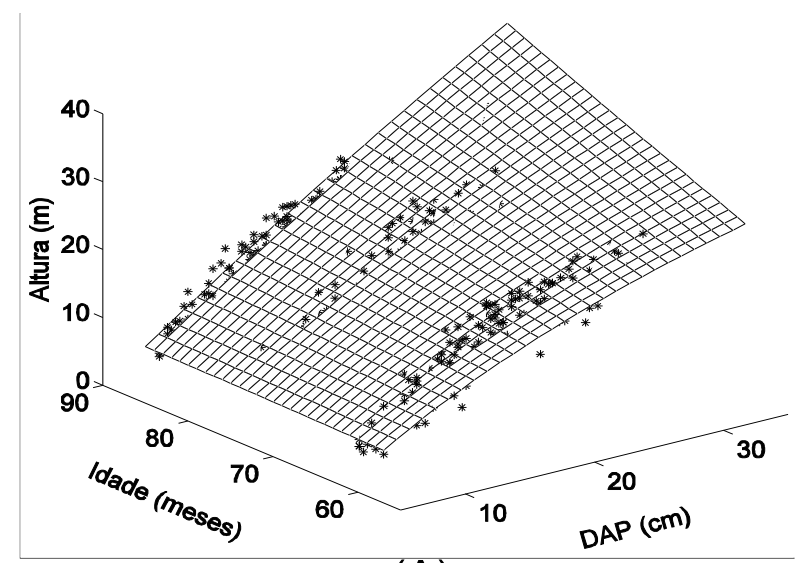

(A)

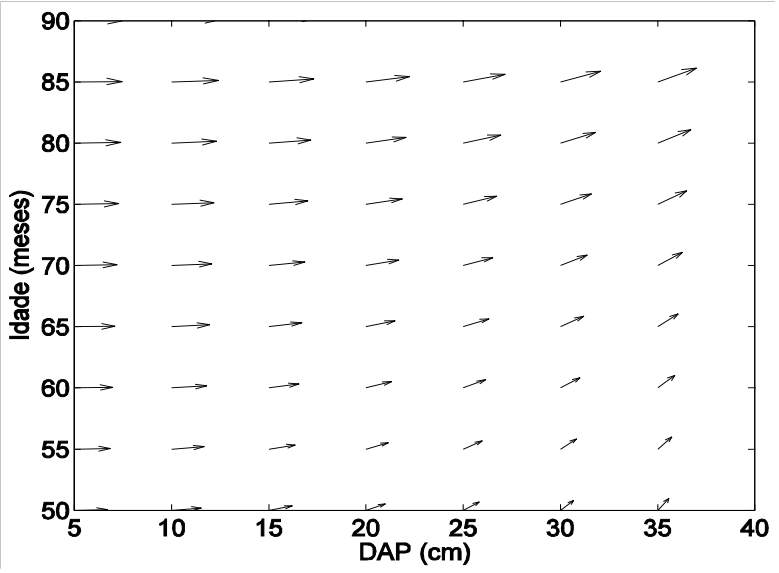

(B)

Figura 4 - Dados do inventário e a superfície da equação de regressão quando considerada todas as árvoresamostra (a); e (b) campo vetorial do fatgradiente de $H, \nabla f(D A P, I d)=f_{D A P}(D A P, I d) \boldsymbol{i}+f_{I d}(D A P$, $I d) j$.

Figure 4-Inventory data and the surface of the regression model when considered all sample-trees (a); and (b) gradient field of $H$ (height),$\nabla f(D A P, I d)=$ $f_{D A P}(D A P, I d) \boldsymbol{i}+f_{I d}(D A P, I d) \boldsymbol{j}$.

Na Figura 5ace são apresentados os $C V s$ da altura estimada das árvores-teste calculados, conforme anteriormente descrito, a partir das 10.000 simulações para cada número de árvores-amostra. Esses resultados foram separados por idade das árvores-teste sendo as letras $(a),(c),(e)$ relativas às idades 60, 74 e 84 meses, respectivamente. Observou-se que para o número de 6 a 18 árvores-amostra para a geração das equações os valores de $C V$ da altura foram altos, chegando a atingir valor superior a $1.000 \%$ (Figura $4 a$ ), ou seja, 
tal tamanho de amostra é visivelmente insuficiente para se gerar uma equação capaz de estimar a altura das árvores com precisão aceitável. A partir de 27 árvoresamostra (9 árvores-amostra de cada idade) para estimar as equações, notou-se queda brusca nos valores do $C V$ da altura das árvores-teste, indicando que esse tamanho de amostra proporciona estimativas da altura bem mais precisas. Para visualizar melhor essa observação, é apresentado na Figura 5bdf o $C V$ da altura das amostrasteste a partir de um número de 27 árvores-amostra para geração das equações de regressão. Pode-se verificar, por exemplo, que nas amostras-teste de árvores de 60 meses o $C V$ calculado a partir de equações geradas com 27 árvores-amostra variou entre $6 \%$ e 10\%, sendo uma variação bem inferior à ocorrida para tamanhos de amostras menores. Outra observação importante e relacionada com o objetivo deste trabalho é que o aumento no tamanho da amostra a partir de uma amostra com 27 árvores não implicou uma queda significativa no $C V$, havendo estabilização mais nítida deste a partir de amostras com 51 árvores. Mesmo assim, embora a taxa de decréscimo do $C V$ para amostras com tamanho variando de 27 a 51 árvores-amostra seja maior, esses valores não excedem a uma diferença superior a aproximadamente $2 \%$, ou seja, um tamanho de amostra com 30 árvores pode apresentar, tomando como base esses resultados, precisão similar à obtida para tamanhos de amostras bem maiores.

Na Figura $6 a b$ são apresentados dois exemplos do comportamento da altura média e desvio-padrão das amostras-teste com 74 meses de idade, calculadas a partir das 10.000 simulações das equações de regressão geradas de 9 e 27 amostras, respectivamente. Assim, cada ponto na Figura $6 a b$ representa a altura média de determinada árvore-teste calculada a partir de 10.000 estimativas obtidas das equações de regressão simuladas para amostras com tamanhos de 9 e 27 árvores, respectivamente. A barra que acompanha cada ponto ou média mede o desvio-padrão em torno desta média. Embora o valor médio calculado para as 10.000 simulações esteja próximo do valor da altura medida em campo para as árvores-teste, nos dois tamanhos de amostra (9 e 27 árvores) o desvio-padrão das estimativas em torno das respectivas médias foi muito diferente entre os dois tamanhos de amostra. As alturas estimadas por equações de regressão das amostras compostas de apenas nove árvores apresentaram desvios-padrão nitidamente superiores àqueles gerados a partir de amostras com 27 árvores.
Como exemplo, uma árvore da amostra-teste cuja altura medida em campo foi de $25,0 \mathrm{~m}$, apresentou altura média estimada pelas 10.000 simulações de $23,7 \mathrm{~m}$ e desvio-padrão de 114,1 m para um tamanho de amostra igual a nove árvores. Quando utilizadas 27 árvores na composição da amostra, a altura média e o desviopadrão foram de 21,6 m e 0,9 m, respectivamente. Esse resultado indica que para o tamanho de amostra maior, correspondente a 27 árvores, as estimativas para as árvores-teste foram, sem dúvida, muito mais precisas, tendo em vista o desvio-padrão calculado para cada árvore-teste, tal como mostrado pelas barras de desvio da Figura $6 a b$. Esse fato foi ainda mais evidente nas árvores de maior $D A P$, corroborando a sensibilidade da variável dependente do modelo hipsométrico avaliado (modelo (1)) à variável $D A P$, como já foi discutido a partir da Figura 4.

Com os resultados apresentados até aqui, podese observar a influência do tamanho e composição da amostra sobre a precisão das equações de regressão ajustadas para o modelo de relação hipsométrica selecionado. Essa preocupação em se estudarem tamanho e composição de amostras para ajuste de uma equação de volume foi demonstrada por Guimarães e Leite (1996), quando esses autores testaram o efeito de diferentes tamanhos de amostra sobre a precisão de uma equação de volume, com a diferença de que eles trabalharam com um número de simulações muito menor. Mesmo assim, os referidos autores concluíram, tal como neste trabalho, que com um número bem menor de árvores-amostra poderia se chegar a uma precisão semelhante para as estimativas geradas quando se trabalhava com um número bem maior de árvoresamostra.

Quando se trabalha, no entanto, com equações de volume, o processo de seleção de árvores-amostra para ajuste das equações normalmente segue um critério em que se procura trabalhar com números iguais de árvores por classe de diâmetro, ou seja, as classes de diâmetro ou de tamanho de árvore são representadas com a mesma intensidade, donde se espera que as estimativas geradas não sejam tendenciosas para nenhuma das classes, dado que elas estão igualmente representadas. O mesmo não ocorre quando se ajustam equações hipsométricas empregando dados oriundos de inventários florestais tradicionais. Os inventários florestais tendem a amostrar mais intensamente os valores médios, fazendo que as estimativas dos valores extremos

R. Árvore, Viçosa-MG, v.31, n.4, p.685-694, 2007 
possam ter algum tipo de tendência. A Tabela 2 ilustra a distribuição de freqüência das 481 árvores-amostra empregadas neste estudo em classes de diâmetro e altura. Conforme mencionado, fica claro, por essa tabela, que as classes intermediárias apresentam freqüência maior de árvores do que as classes extremas, comprovando

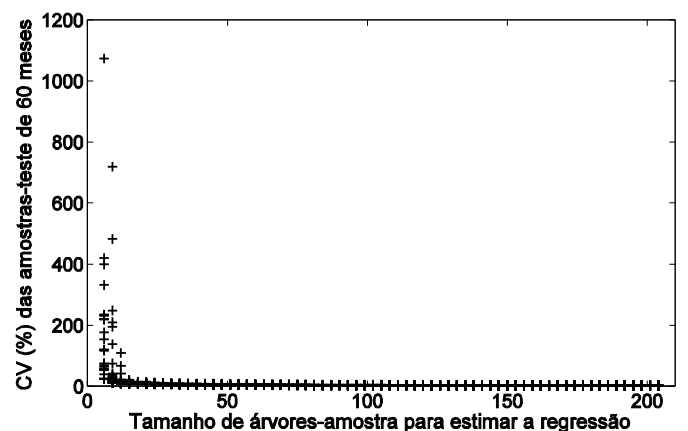

(a)

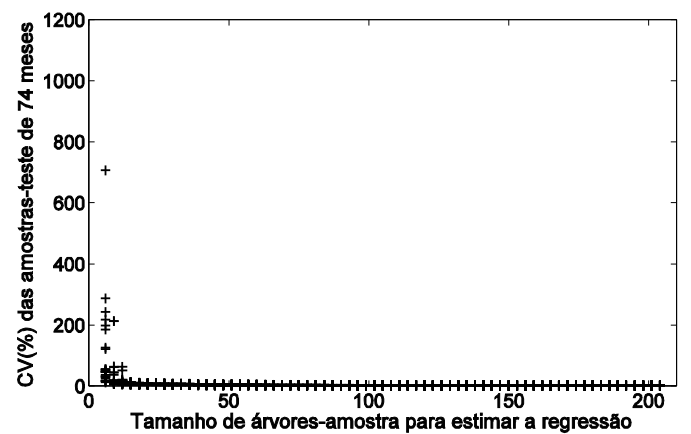

(c)

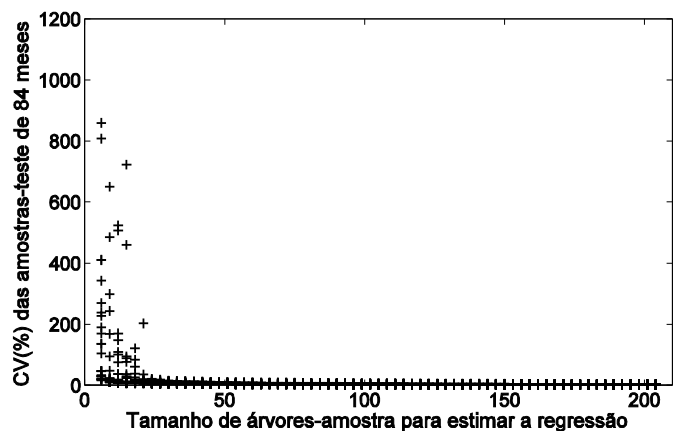

(e) a tendência dos inventários florestais tradicionais em amostrarem mais intensamente os valores médios. Assim, na composição das amostras para ajuste das equações de regressão as classes extremas foram bem menos representadas, não se esperando, portanto, muita precisão nas estimativas das árvores pertencentes a essas classes.

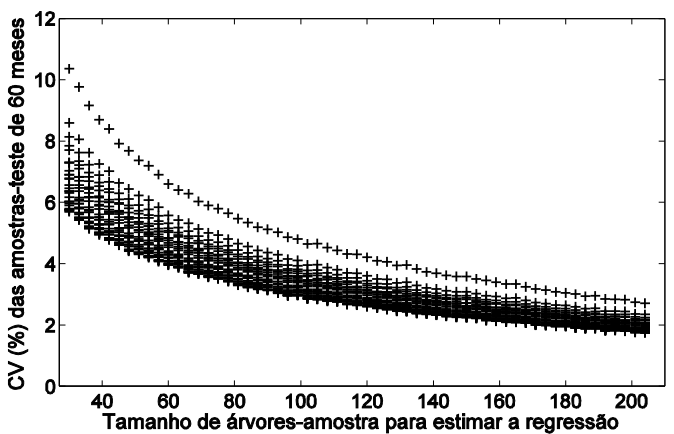

(b)

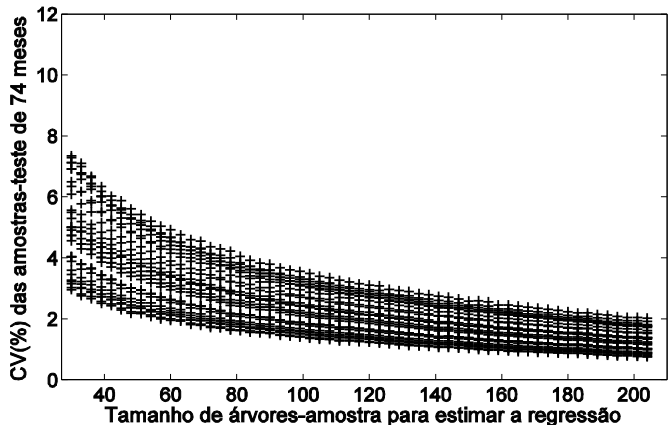

(d)

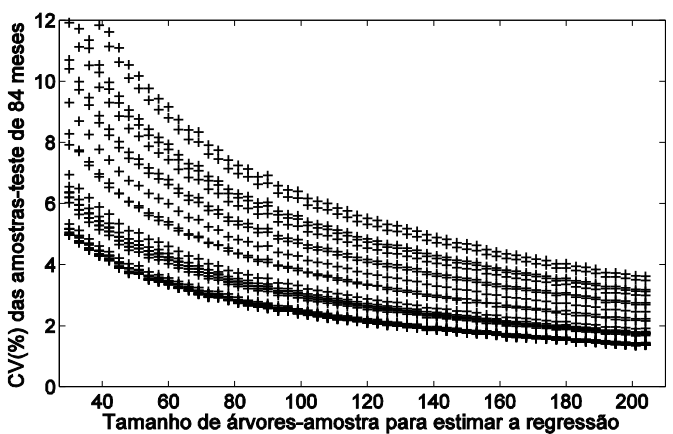

(f)

Figura 5 - Relação entre os coeficientes de variação da altura estimada das árvores-teste calculados a partir das 10.000 simulações e o número de árvores-amostra considerando-se as diferentes idades. As letras $(a),(c)$ and $(e)$ são relativas às idades 60, 74 e 84 meses, respectivamente, para tamanho de amostras variando de 6 a 204 árvores, e as letras $(b),(d),(f)$ são relativas às idades 60, 74 e 84 meses, respectivamente, para tamanho de amostras variando de 27 a 204 árvores.

Figure 5 - Ratio between variation coefficients of estimated test-tree height calculated from 10,000 simulations and the number of sample-trees considering the different ages. Letters $(a),(c)$ and (e) refer to the ages 60, 74 and 84 month, respectively, for the sample size varying from 6 to 203 samples, and the letters $(b),(d)$ and $(f)$ also refer to the tree age of 60, 74 and 84, respectively, but with sample size varying from 27 to 204.

R. Árvore, Viçosa-MG, v.31, n.4, p.685-694, 2007 

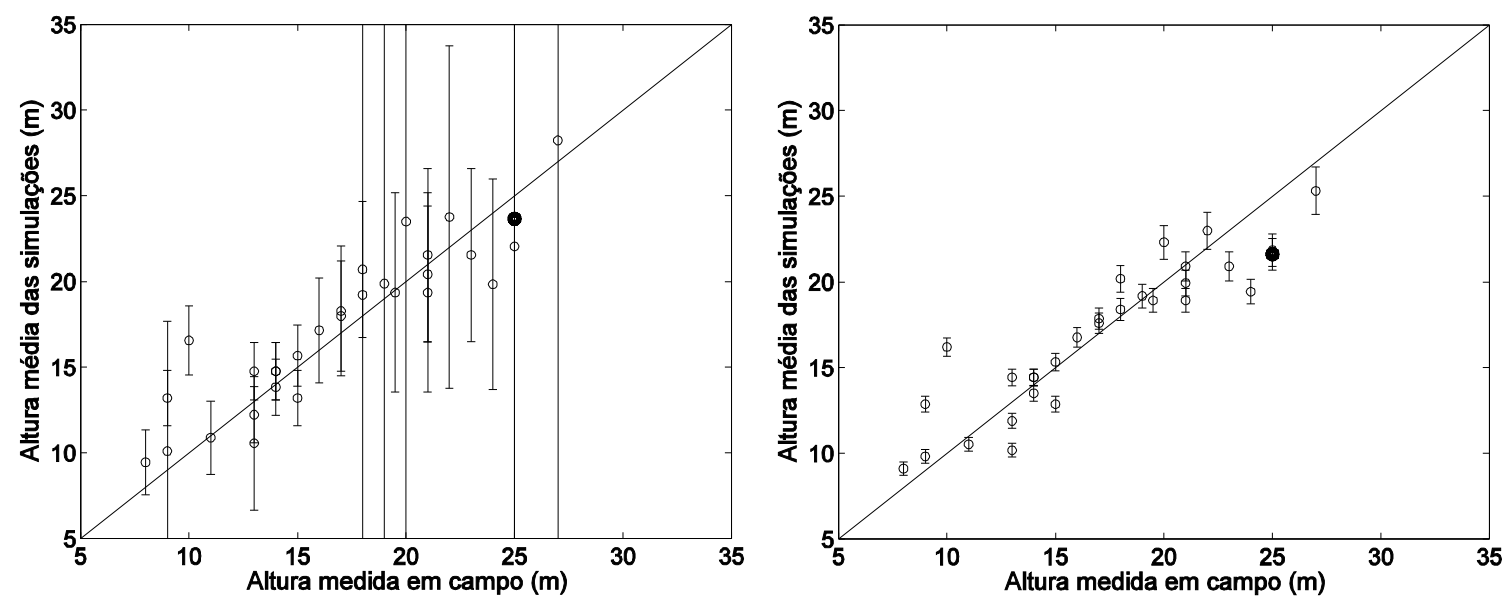

Figura 6 - Relação entre a altura medida em campo e a altura média das simulações das 30 amostras-teste aos 74 meses de idade, considerando-se tamanhos de amostra de $9(a)$ e $27(b)$ árvores-amostra para o ajuste da equação de regressão.

Figure 6 -Ratio between height taken in the field and mean height of simulations for 30 test-samples of 74 month-old trees, considering sample size of $9(a)$ and $27(b)$ tree-samples to ajust the regression equation.

Considerando o exposto, além do tamanho da amostra, a sua composição é muito importante para que as equações de regressão representantes das relações hipsométricas proporcionem estimativas mais confiáveis. Nesse sentido, ficou claro neste estudo de caso que o procedimento de inventário aqui apresentado, em que foram medidos os pares de diâmetro e altura das 18 primeiras árvores de todas as parcelas, poderia ser substituído por um procedimento onde se poderia medir um número menor de árvores que deveriam ser escolhidas seletivamente, de modo a representar todas as classes de diâmetro, sem que houvesse, no final, prejuízos significativos em termos de perda de precisão nas estimativas das alturas das árvores.

Mesmo não tendo sido objetivo deste trabalho, é importante mencionar que em inventários florestais, além da precisão, avaliar o custo e operacionalidade dos procedimentos é fundamental para que se consiga um resultado final satisfatório. Assim, seria objeto de interesse em estudos posteriores a avaliação de tempos de coleta dos dados, bem como seus custos associados, de modo a avançar nas conclusões para se obterem métodos de inventários precisos, operacionais e de baixo custo.

\section{CONCLUSÕES}

Considerando todos os resultados encontrados, chegou-se às seguintes conclusões:
$\Rightarrow$ Neste estudo de caso envolvendo a espécie Eucalyptus grandis, amostras com pelo menos 27 árvores (9 para cada grupo de idade) proporcionaram estimativas mais precisas da altura das árvores quando comparadas com amostras menores.

$\Rightarrow$ Amostras com tamanhos superiores a 27 árvores não proporcionaram ganhos significativos, em termos de aumento de precisão, na estimação das alturas das árvores.

$\Rightarrow$ A variável $D A P$ foi mais importante para estimar a altura do que a variável idade, considerando-se o modelo (1) proposto. Cabe ressaltar que a influência da variável idade na estimação da altura foi maior nas árvores de maior $D A P$.

\section{REFERÊNCIAS}

BATISTA, J. L. F.; COUTO, H. T. Z.;

MARQUESINI, M. Desempenho de modelos de relações hipsométricas: estudo em três tipos de florestas. Revista do IPEF, v.1, p.33-44, 1986.

COUTO, H. T. Z.; BASTOS, N. L. M. Modelos de equações de volume e relações hipsométricas para plantações de Eucalyptus no Estado de São Paulo. Scientia Forestalis, n.60, p.149-163, 1986. 
FINGER, C. A. G. Fundamentos de biometria florestal. Santa Maria: Universidade Federal de Santa Maria, Centro de Pesquisas Florestais, 1992. 269p.

GUIMARÃES, D. P.; LEITE, H. G. Influência do número de árvores na determinação de equação volumétrica para Eucalyptus grandis. Scientia Forestalis, n.50, p.37-42, 1996.

LOETSCH, F.; ZOEHRER, F.; HALLER, K. E. Forest inventory. Munchen: B VL, 1973. v.2. 469p.
MATHWORKS, Inc. MATLAB, The language of technical computing. Natick: 1996.

SCHINDLER, P. R. Manejo florestal I Tópicos para o planejamento da produção florestal. Santa Maria: Universidade Federal de Snata Maria, 1986. 289p. (Apostila).

\section{SCOLFORO, J. R. S. Mensuração}

florestal 3: relações quantitativas: em volume, peso e a relação hipsométrica. Lavras: DCF-ESAL, 1993. 291p. 